\title{
La práctica docente de enfermería en "educación para la salud"
}

\author{
Araceli Sánchez Ramos
}

\section{RESUMEN}

El presente ensayo aborda la siempre cambiante enseñanza de una asignatura del currículo básico enfermero. Trata de profundizar en el conocimiento de la educación para la salud, concretamente en el marco de enseñanza de enfermería, y aportar así una propuesta educativa de la misma, que se pueda concretar en un programa de formación en la educación para la salud (EpS), acorde con las necesidades reales de la sociedad actual. La educación para la salud está considerada en la actualidad, como uno de los instrumentos básicos para poder ejercer las funciones de promoción de la salud y prevención de enfermedades, acciones básicas en torno a problemas de salud; es por todo esto, que se debe prestar mayor atención a todos aquellos aspectos que supongan una mejora y perfeccionamiento del quehacer en el aula, que redunde en una optimización de la calidad de los cuidados enfermeros que se prestan en la comunidad. Objetivo: obtener una aproximación concreta sobre los factores influyentes en la enseñanza de la Educación para la Salud en el seno de las escuelas de enfermería; así como reflexionar sobre la entidad propia de cada disciplina en este caso la educación para la salud. Analizar su enseñanza, captada y comprendida por profesionales enfermeros.

Palabras clave: educación para la salud, práctica docente de enfermería, enseñanza.

\section{Teaching nursing practice in "health education"}

\begin{abstract}
This test addresses always changing teaching of a subject of the basic curriculum nurse. This is knowledge of the health, specifically in the framework of the school of nursing, education and to provide an educational proposal, to be specified in a training programmed in education for health, (EpS) meets the real needs of today's society. Education for the health is considered as one of the core to exercise the functions of promoting health and preventing disease, Basic actions on health; instruments is now by all this, we should provide more attention to all aspects involving an improvement and refinement of the work in the classroom, which is in the optimisation of the quality of care provided by the community nurses. The main objective of this test is to obtain a specific approach on the factors influencing the health within the school of nursing education teaching as well as to reflect on the entity itself in each discipline in our case the health education. Analyzes his teaching.
\end{abstract}

Key words: education for health, practical nursing, teaching education.

Correspondencia: Araceli Sánchez Ramos. Subdirección de Investigación en Enfermería. Instituto Nacional de Neurología y Neurocirugía. Insurgentes Sur \# 3877. Col. La Fama 14269 México, D.F. E-mail: celisanch@yahoo.com.mx

I

os problemas que en la actualidad afectan la salud humana; son una preocupación de investigadores y científicos; tanto en México como del mundo, los mismos constituyen objeto de análisis y valoración por la repercusión que tienen para la vida del hombre en el planeta. Para enfrentar estos problemas no debe predominar la labor aislada pues en ella deben colaborar los distintos factores de la sociedad.

En este sentido las escuelas de educación plantean direcciones de trabajo y programas con enfoque eminentemente pedagógico, por lo cual los educadores no están ajenos a estos problemas y se les convoca a trabajar en aras de educación sanitaria que complementen los servicios que se brindan por los profesores durante la labor educativa, con el objetivo de prevenir y promover la salud del escolar para ello es necesario que se mantengan actualizados en los temas y adelantos de la salud, fundamentalmente de la higiene y epidemiología en virtud que se preparen para ejercer una educación para la salud más efectiva. 
Por ello; es imperativo ofrecer variadas oportunidades, entornos y ambientes saludables tanto, en escuelas de enfermería como de la comunidad para involucrarse en un proceso de aprender haciendo, donde puedan utilizar su creatividad y entusiasmo para lograr participación plena en el desarrollo de actitudes crítico-reflexivas frente a las prácticas, y factores de riesgo, lo cual exige preparar al personal pedagógico en formación. Aún cuando los esfuerzos realizados en el orden material y técnico muestran avances, subsisten problemas que son necesarios resolver en un plazo breve para contribuir a fomentar una cultura para la salud. Esto es posible si se tienen sólidos y profundos conocimientos y si se establece buena comunicación entre estudiantes de enfermería, comunidad y otros factores de la sociedad, de forma que se logre influir positivamente, involucrando activamente en esta tarea a todos.

La educación para la salud esta considerada en la actualidad, como uno de los instrumentos básicos para poder ejercer funciones de promoción y prevención de enfermedades; así como, acciones en torno a los problemas de salud; es por todo esto, que hay que presentar mayor atención a todos aquellos aspectos que suponen una mejora y perfeccionamiento del quehacer en el aula, que redunde en una optimización de la calidad de los cuidados enfermeros que se prestan en la comunidad.

\section{La OMS y la práctica de educación para la salud}

El estudio que la Organización Mundial de la Salud (OMS) realizó en 1996, sobre las reformas sanitarias en México, evidenciaron que la enfermería es el primer eslabón y el punto de contacto más sólido, entre el sistema y las personas, por consiguiente en la naturaleza de la enfermería, se conjuga el ser disciplina profesional en cuanto a una práctica social, cuya misión es cuidado de salud del ser humano individual y colectivo a través de los procesos vitales y en contextos culturales específicos para alcanzar mejores condiciones de $v i d a^{1}$. Su acción está orientada por principios y fundamentos científicos, humanísticos y éticos de respeto a la vida y dignidad humana. Su práctica requiere de pensamiento interdisciplinario, actuar multiprofesional, y un equipo con visión holística del ser humano en su relación con el contexto social y ambiental.

Esto legitima a la enfermería, como elemento clave en el engranaje entre padres, docentes, alumnos y profesionales de la salud ${ }^{2}$.

\section{Evolución de enfermería hacía el primer nivel de atención a la salud}

Hasta 1970, la formación de recursos humanos para enfermería, acorde con las demandas del mercado de trabajo, estaban encaminados a capacitar a los profesionales de enfermería, para responder a las necesidades de salud de la población atendidas en Instituciones públicas o privadas de segundo y tercer nivel.

Si bien, existió el trabajo de enfermería en las comunidades (enfermera sanitarista) está no era una práctica dominante; pues su preparación se inclinaba más a la especialización, esto les permitió (a los profesionales de enfermería) funcionar mejor en los hospitales de tercer nivel, donde existía una mayor demanda, y podían realizarse profesionalmente, con un mejor estatus económico y social.

A partir de 1980, la nueva política de salud propone estratificar la atención de la salud por niveles, con énfasis en el primer nivel de atención. Por lo tanto, las escuelas de enfermería deben enfocar en sus planes de estudio la formación de estudiantes con significado especial en promoción a la salud y realizar cambios en la formación del recurso humano, para dar respuesta a las políticas de salud; así como, a los problemas detectados en el panorama epidemiológico de la salud. Por ello se propone que en los planes de estudio incluyan entre otros aspectos los siguientes objetivos:

Adquisición de las herramientas teórico-metodológicas que le permitan desarrollar habilidades y actitudes para:

- Analizar el panorama epidemiológico del país a través del modelo social en el que está inmersa la población.

- Identificar cuales son las causas sociales que actúan como factores de riesgo en el panorama epidemiológico de México.

- Participar con el equipo de salud, en investigación y vigilancia epidemiológica de problemas de salud que más afectan a los diferentes grupos sociales según edad y sexo.

- Formular programas de educación para la salud dirigidos a la comunidad para disminuir factores de riesgo, fomentando participación del grupo familiar, diferentes grupos sociales y de individuos en lo particular en el autocuidado de la salud ${ }^{3 .}$

\section{Escuelas de enfermería y educación para la salud}

La escuela es una institución llamada a participar en la EpS, sus características abren posibilidades de logros en su acción; los requisitos de asistencia para los involucrados y las posibilidades de espacios y materiales contribuyen a los programas de salud en las escuelas para que vayan más allá de la mera difusión, e información y que ayuden a los estudiantes a la adquisición de conocimientos para ayudar a la población a tener hábitos saludables.

Si se pretende que las nuevas generaciones adquieran valores, conocimientos y prácticas indispensables para preservar y mejorar la salud, es necesario que la escuela promueva 
actividades creadoras, debidamente planeadas, exigiendo la intervención de todos los partícipes del proceso educativo y respondiendo a las características del contexto social en el que está inmersa la escuela.

La educación para la salud necesita, un proceso dinámico que les de aliento y ayude a desempeñar funciones que corresponden, en esa acción en la que todos deben unir sus fuerzas y voluntad.

Tradicionalmente, en muchas escuelas la enseñanza de la salud está fragmentada en multitud de conocimientos que se imparten con otras materias. En algunos sistemas la EpS se considera una materia distinta o separada de las demás. La escuela es responsable de diseñar estratégias que respondan a condiciones reales en las que se desarrolla el alumno y en donde él pueda atribuir un significado a los conocimientos adquiridos.

Para que los profesionales de enfermería puedan convertirse en verdaderos promotores de la salud, los profesores deben partir de un conocimiento de los grupos sociales que la conforman, de sus problemas educativos, sus carencias y sus necesidades, pues no se podría desarrollar un programa en salud, si no se parte de un diagnóstico que oriente adecuadamente la intervención ${ }^{4}$.

\section{La enseñanza de enfermería en educación para la salud}

La enseñanza de enfermería en educación para la salud, ha centrado su atención en aspectos que se consideran más reelevantes, en términos de los cambios que son necesarios para que se lleven a cabo, se han analizado programas actuales de formación de enfermeras en México y América Latina, a la luz de la problemática de salud de enfermería, tendencias y su significado para la práctica de enfermería de salud comunitaria. Considerando los conceptos básicos adoptados, se han identificado ciertos aspectos de capacitación de las enfermeras que deberían ser modificados o enfocados de manera distinta a fin de que las egresadas puedan adquirir una filosofía orientada hacia el trabajo en comunidad ${ }^{5}$. Esta orientación de acuerdo con Sidney Kark; considera primordialmente la necesidad de estimar y anticipar los problemas individuales de salud a nivel de la comunidad, y no se limita a problemas de personas y grupos que buscan atención de salud. Además, demanda vigilancia continua del sistema de salud que tiene en cuenta el tipo de servicio prestado, a quiénes se presta este, en qué etapa de la historia natural del problema se ubica, cuando es posible evaluar la relación entre los servicios prestados, y los cambios logrados en la salud de grupos atendidos y en indicadores generales de salud de la población $n^{6}$.

\section{CONCLUSIONES}

No es sencillo tratar de definir el papel de la práctica docente como promotora de la salud, el desarrollo de este ensayo pretende señalar algunas líneas generales para reflexionar sobre posibles caminos que nos conduzcan a todos a vivir en mejores condiciones. La definición de etapas de cambio, reestructuración de conceptos, colaboración de integrantes de un equipo multidisciplinario, organización de contenidos, planificación de actividades, de quienes estamos interesados en heredar a las futuras generaciones una vida digna y llena de salud, esta enfocada a contribuir en educar en materia de salud desde el inicio de la carrera de enfermería. Se debe enfrentar el reto reflexionando sobre la formación de docentes, aciertos y desaciertos de nuestra práctica profesional para afrontar la tarea con las armas suficientes que nos proporcionen diversas disciplinas.

Los múltiples problemas de salud que afectan en la actualidad a la humanidad, demandan una intervención educadora. Los profesores, desde las aulas, deben buscar alternativas para contribuir en la práctica docente de educación para la salud y así favorecer a la construcción de un mundo mejor, en el que todos los seres humanos tengan una calidad de vida mejor.

\section{REFERENCIAS}

1. Flores Bienert M, Armero Barranco D, García Herrero M. La enseñanza en la educación para la salud vista por profesionales enfermeros de atención primaria de salud. www.imss.gob.mx/NR/ rdonlyres/71C3EFC1-AB15.../6163.pdf Consultado 11 de septiembre 2009 22:00 hs.

2. Fernández Candela D, Rebolledo Malpica M, Velandia Mora A. Hacia la promoción de la salud. Volumen 11, enero-diciembre 2006.

3. Domínguez Hernández G. La profesión de enfermería en el trabajo comunitario www.imss.gob.mx/NR/rdonlyres/71C3EFC1-AB15.../ 6163.pdf consultado el 11 de septiembre 2009 22:00 hs.

4. Álvarez Alba R. Educación para la salud. Manual Moderno, México 1998.

5. Atención primaria a la salud. docentes.uacj.mx/.../introduccion\% 20al\%20estudio\%20de\%20la\%20salud\%20publica/Consultado 14 de Septiembre 2009- 24:08 hs.

6. Leavell HR, EG Clark. Preventive medicine for the doctor in his community. An epidemiological approach. 3a edición. Nueva York. McGraw-Hill Book Co. 1965. 